\title{
New optogenetics technique minimizes thermal damage to neurons
}

\author{
BY NIKO MCCARTY
}

8 JULY 2021

A new engineered protein for optogenetics experiments requires less light than traditional techniques to excite or inactivate neurons, reducing thermal damage to brain tissue.

The protein, described in eLife in May, improves a method called two-photon optogenetics, which enables researchers to study neural circuits by stimulating them and simultaneously monitoring their activity, including in living mice. It could help researchers map the neural circuits that connect different autism-linked brain regions, such as the amygdala, prefrontal cortex and cerebellum.

To employ two-photon optogenetics, scientists first engineer cells in an animal's brain to express two proteins: an opsin, which activates or silences neurons in response to pulses of blue light, and a calcium sensor, which fluoresces red light when it binds to calcium ions; these ions flow into a neuron just before it fires. Researchers can then use two separate lasers to stimulate a programmed series of individual neurons and then monitor their activity levels. By contrast, traditional optogenetics relies on LED lights to switch on thousands of neurons at once.

"By stimulating individual neurons, we can start actually asking questions about information encoding in the brain in a way that's impossible with wide-field, single-photon optogenetics," says Ofer Yizhar, professor of neurobiology at the Weizmann Institute of Science, Israel, who devised the novel protein.

Previous proteins used in two-photon optogenetics often have spectral overlap, which means that the red laser can inadvertently activate the light-sensitive opsins. And some of the opsin proteins have presented another challenge: They require heavy pulses of light to activate, which can heat up and damage brain tissue.

\section{Less light:}




\section{Spectrum | Autism Research News}

https://www.spectrumnews.org

Yizhar and his colleagues made a modified version of an existing opsin protein by attaching a short peptide sequence that directs it to the soma, or cell body, of neurons, and a fluorescent tag, which helps the team visualize engineered cells. The original protein, CoChR, is expressed throughout a neuron's dendrites and axons, making it challenging to detect and target. They call the new protein stCoChR, or 'soma-targeted channel rhodopsin.'

The team engineered mice to express stCoChR in pyramidal neurons in the cerebral cortex, with or without an existing calcium sensor called jRCaMP1a.

The researchers also developed and tested a laser system that can deliver more energy to neurons per pulse than typical lasers, but at a lower repetition rate. The energy required to stimulate neurons fell well below the threshold that causes damage to brain tissue, they found. The engineered opsin could also be stimulated more efficiently than prior versions and had no spectral overlap with the calcium sensor.

"Now that we have this channel rhodopsin that's really sensitive, we can turn down the light power," Yizhar says. "This means that we can split the laser up into multiple beamlets, and we can now have 100-fold more beamlets to stimulate 100-fold more neurons, which gives us much better flexibility to generate pretty much any pattern that we want to stimulate."

The technique has some limitations: Calcium sensors are an indirect measure of neural activity, so the laser cannot capture every action potential. Yizhar says he hopes to use voltage indicators proteins that directly sense electrical charges in neurons - in future experiments.

With the improved opsin protein, he and his colleagues also plan to dissect the neural circuits underlying learning and autism.

"We're interested in the prefrontal cortex and in autism models of neural connectivity," Yizhar says. "The kind of questions that we're interested in are how the prefrontal network is wired up, how it changes in the process of learning and also how it changes as a result of autism-related mutations."

Cite this article: https://doi.org/10.53053/IGVE9122 